\title{
Renal Involvement in Leprosy: Retrospective Analysis of 461 Cases in Brazil
}

\author{
Geraldo Bezerra da Silva Júnior \\ and Elizabeth De Francesco Daher
}

\author{
Department of Internal Medicine, School of Medicine, Federal \\ University of Ceará, Fortaleza, CE, Brazil
}

\begin{abstract}
Background. Renal lesions in leprosy have been extensively described, including amyloidosis, glomerulonephritis, nephrosclerosis, tubulointerstitial nephritis and granulomas. Material and Methods. A retrospective study was designed to detect renal function abnormalities in 461 leprosy patients, without any co-morbidity, seen in a university hospital in northeast Brazil. The laboratory test results concerning renal function were examined in the patients' medical records. Results. The mean age was $39 \pm 18$ years and $217(47 \%)$ were male. The mean duration of disease was $21 \pm 38$ months. Levels of creatinine above $1.4 \mathrm{mg} / \mathrm{dL}$ had been detected in 40 patients $(8.6 \%)$. The levels of creatinine and urea were higher in lepromatous leprosy patients. Proteinuria, hematuria and hemoglobinuria were significantly more frequent in this presentation of leprosy. Nephrotic levels of proteinuria $(>3.5 \mathrm{mg} / \mathrm{dL}$ ) were found in three $(\mathbf{2 . 1 \%})$ of 138 lepromatous leprosy patients. A positive association was found between duration of disease and high levels of creatinine in the general sample and in the lepromatous leprosy patients. There was no association between time of treatment and high levels of creatinine.Conclusion. Renal involvement in leprosy seems to be related to the quantity of bacilli present in the body. It is important to evaluate the renal function in all leprosy patients in order to detect any abnormality and prevent renal failure, which is still a potential cause of death in this disease.
\end{abstract}

Key Words: Leprosy, kidney, renal abnormalities, renal failure.

Leprosy is still an important public-health problem. Brazil is the second country in the world in number of cases [1,2]. Renal abnormalities in leprosy have been widely described in the medical literature [3-11]. Mitsuda \& Ogawa [12] were the first to report renal lesions in this disease, through autopsy findings. Kean \& Childress [13] described glomerulopathies, tubulopathies and nefrosclerosis in the kidneys of leprosy patients, also through autopsy findings. Renal injury seems to be common in patients with erythema nodosum leprosum (ENL) [8]. In a review of 199 autopsies of Brazilian leprosy patients, renal lesions were found in 144 (72\%) of them; they presented amyloidosis, glomerulonephritis, nephrosclerosis, tubulointerstitial nephritis, granulomas, and other lesions [8].

Glomeruli injury has been described in histology findings in leprosy patients, progressive mesangial glomerulonephritis being the most common lesion [7,8,14]. Many other kinds of glomerulonephritis have also been described [3$6,8,10,11,13,15,16]$. The incidence of glomerulonephritis has been reported to range from $6 \%$ to $50 \%$ in leprosy patients [17]. Amyloidosis, the incidence of which ranges from 2\% to 55\% [17], is attributed to chronic granolomatous reactions caused by Mycobacterium leprae [18], and it is manifested mainly by significant proteinuria [19]. It may progress to chronic renal failure, which is one of the causes of death in leprosy [20].

Received on 16 October 2005; revised 22 February 2006.

Address for correspondence: Dr. Geraldo Bezerra da Silva Júnior. Rua 25 de março, 997. CEP: 60060-120. Fortaleza, CE, Brazil. Phone/ Fax: +55 85 3273-2445 / +55 85 3261-3777.

E-mail: geraldobezerrajr@yahoo.com.br,ef.daher@uol.com.br.

The Brazilian Journal of Infectious Diseases 2006;10(2):107-112. (C) 2006 by The Brazilian Journal of Infectious Diseases and Contexto Publishing. All rights reserved.
The exact pathogenesis of renal lesions in leprosy is still uncertain [18]. The bacteria do not seem to be directly involved in the renal lesions [12], although they have been detected in the glomeruli and renal parenchyma of some patients $[8,14]$. The glomerular lesion is probably caused by immunecomplexes, which develop during the reactional states, mainly in erythema nodosum leprosum ${ }^{18}$. This is supported by clinical and laboratorial findings, such as the visualization of immunecomplexes and a decrease of serum complement in some cases [5,7,21-25].

After approval by the Ethics Committee of the Walter Cantídio University Hospital, Faculty of Medicine, Federal University of Ceará, Brazil, we carried out a retrospective study in order to detect renal function abnormalities in leprosy patients through analysis of clinical and laboratorial data from the medical records.

\section{Material and Methods}

The patients eligible for inclusion in this study were all those with confirmed diagnosis of leprosy admitted to or seen in the Walter Cantídio University Hospital, Faculty of Medicine, Federal University of Ceará, Brazil, in the period between January 1976 and December 2002. Patients were excluded if they had any co-morbidity, such as hypertension, diabetes, urinary tract infection, or any other conditions that could be responsible for renal dysfunction.

Diagnosis had been made based on clinical and laboratorial findings, such as skin lesions, peripheral nerve injury, bacilloscopy and histopathological analysis of skin lesions. The patients were classified according to the recommendations of the World Health Organization, as indeterminate (I), tuberculoid (TT), borderline tuberculoid (BT), 
mid-borderline (BB), borderline lepromatous (BL) and lepromatous (LL).

A medical record with the following items was made for each patient: identification (name, age, gender, job, origin, address, telephone number), time of disease, time of treatment, symptoms and signs, laboratorial finds during treatment (serum urea and creatinine, serum sodium and potassium and urinalysis).

The means of the maximum values of serum urea and creatinine found during the course of the disease were calculated. Proteinuria, hematuria, hemoglobinuria and leukocyturia were analyzed through the use of reagent strips. We considered proteinuria and hemoglobinuria as one or more "+" in the qualitative exam. We considered levels of protein above $3.5 \mathrm{~g} / \mathrm{dL}$ in $24 \mathrm{~h}$ proteinuria as nephrotic. Hematuria was considered as one or more " + " in the qualitative exam and one or more erythrocytes per high power field. Leukocyturia was considered as the presence of five or more leukocytes per high-power field.

Renal failure was defined as a serum level of creatinine $\geq 1.4 \mathrm{mg} / \mathrm{dL}$. We compared the results from tuberculoid leprosy patients with those from lepromatous leprosy patients, which represent the two poles of the disease, in order to determine whether renal abnormalities are more commonly associated with the multibacillary form of the disease. An analysis was also made of time of disease, time of treatment and high levels of creatinine to determine if these variables are associated.

The results were expressed as mean \pm SD. All data were analyzed through the programs Epi Info, version 6.04b 2001, and Stata, version 7.0 2001. The t test and Fischer's exact test were performed to verify the levels of significance in the differences between the data from tuberculoid leprosy and lepromatous leprosy patients. The chi square test was used to determine if there was association between duration of disease, duration of treatment, age, and high levels of serum creatinine. A 5\% $\mathrm{P}$ value or an odds ratio confidence interval of $95 \%$, were considered significant.

\section{Results}

During the period of this study, 547 inpatients and outpatients with leprosy were attended at the Walter Cantídio University Hospital, 461 of which had no co-morbidity; 217 $(47 \%)$ were male and the mean age was $39 \pm 18$ years (range $4-$ 87 ). The mean time of disease was $21 \pm 38$ months (range 1393 ), and the mean time of treatment was $14 \pm 14$ months (range 1-120).

Among these patients, $418(90 \%)$ had used the correct treatment. It consisted of multidrug therapy, as recommended by the World Health Organization, with rifampicin and dapsone for paucibacillary patients and rifampicin, dapsone and clofazimine for multibacillary patients [26,27]. The reactional states were treated with corticosteroids or thalidomide.
According to the WHO classification, the patients were indeterminate (18\%), tuberculoid (28\%), borderline tuberculoid $(11 \%)$, mid-borderline $(4 \%)$, borderline lepromatous $(8 \%)$ or lepromatous $(30 \%)$. Cases of neural leprosy were also found $(1 \%)$. The clinical and epidemiological data of these patients are summarized in Table 1.

Urinary abnormalities were found in all clinical presentations of leprosy. Proteinuria was found in 36 cases $(7.8 \%)$, hematuria in $60(13 \%)$, hemoglobinuria in $34(7.3 \%)$ and leukocyturia in $76(16.4 \%)$. Nephrotic levels of proteinuria $(>3.5 \mathrm{~g} / \mathrm{dL})$ were found in four patients $(0.8 \%$, Table 2$)$.

The mean serum urea and creatinine concentrations of the 461 patients were $91 \pm 71 \mathrm{mg} / \mathrm{dL}$ and $4.1 \pm 5 \mathrm{mg} / \mathrm{dL}$, respectively. Levels of creatinine above $1.4 \mathrm{mg} / \mathrm{dL}$ were detected in 40 patients (8.6\%) and levels of urea above $40 \mathrm{mg} / \mathrm{dL}$ in 52 (11.2\%). Among the patients with renal failure, $18(45 \%)$ were lepromatous, 7 (17.5\%) tuberculoid, $7(17.5 \%)$ indeterminate, $3(7.5 \%)$ borderline lepromatous, $3(7.5 \%)$ borderline tuberculoid and $2(5 \%)$ midborderline. The mean levels of serum sodium and potassium were within the normal values (mean of $135 \pm 5.7 \mathrm{mEq} / \mathrm{L}$ and 4.4 $\pm 0.8 \mathrm{mEq} / \mathrm{L}$, respectively). Electrolytes abnormalities were found in few patients (Table 3). Urinalysis detected proteinuria in 11 of the patients with renal failure (27.5\%), hematuria in $15(37.5 \%)$, hemoglobinuria in $12(30 \%)$ and leukocyturia in $10(25 \%)$. Nephrotic levels of proteinuria $(>3.5 \mathrm{~g} / \mathrm{dL})$ were found in one of these patients $(2.5 \%)$.

A comparison between the patients with tuberculoid leprosy and lepromatous leprosy can be seen in Table 4 . There were significant differences in gender, time of disease, serum levels of urea and creatinine, frequency of renal failure, proteinuria and hematuria. Duration of disease was longer in the lepromatous leprosy patients. Renal failure (creatinine levels $\geq 1.4 \mathrm{mg} / \mathrm{dL}$ ) was more frequent in the lepromatous patients. Abnormalities in the urinalysis (proteinuria, hematuria and hemoglobinuria) were also more frequent in the lepromatous leprosy patients. Leukocyturia was found in both groups, without significant differences. Nephrotic levels of proteinuria $(>3.5 \mathrm{~g} / \mathrm{dL})$ were found in $3(2.1 \%)$ of the lepromatous leprosy patients.

A positive association was found between duration of disease and renal failure in the general sample. The duration of treatment did not show any association with renal failure. Among the tuberculoid leprosy patients $(n=129)$ there was no association between time of disease, time of treatment and renal failure (Table 5).

There were three deaths among the 461 patients, the estimated mortality rate being $0.6 \%$. The causes of death were septic shock, secondary to skin lesion infection, and pulmonary embolism.

\section{Discussion}

The analysis of the epidemiological data shows that leprosy can occur at any age, the development of disease 
Table 1. Clinical and epidemiological data of 461 leprosy patients

\begin{tabular}{lc}
\hline & N (\%) / mean \pm SD \\
\hline Gender & \\
$\quad$ Male & $217(47 \%)$ \\
Female & $244(53 \%)$ \\
AGE (years) & $4-87(39 \pm 18)$ \\
Time of disease (months) & $0-393(20 \pm 38)$ \\
Time od treatment (months) & $0-120(14 \pm 14)$ \\
Forms of leprosy (WHO) & \\
Indeterminate & $85(18 \%)$ \\
Tuberculoid & $129(28 \%)$ \\
$\quad$ Borderline tuberculoid & $49(11 \%)$ \\
Borderline & $19(4 \%)$ \\
Borderline lepromatous & $37(8 \%)$ \\
Lepromatous & $138(30 \%)$ \\
$\quad$ Neural & $4(1 \%)$ \\
\hline
\end{tabular}

Mean $=$ arithmetic mean; $\mathrm{SD}=$ standard deviation. $\mathrm{WHO}-$ World Health Organization criteria.

Table 2. Renal abnormalities found in 461 leprosy patients

\begin{tabular}{lcr}
\hline & $\mathbf{N}$ & $\%$ \\
\hline Blood & & \\
$\quad$ Creatinine $\geq 1.4 \mathrm{mg} / \mathrm{dL}$ & 40 & 8.6 \\
Urea $\geq 40 \mathrm{mg} / \mathrm{dL}$ & 52 & 11.2 \\
Urine & & \\
Proteinuria & 36 & 7.8 \\
Nephrotic levels of proteinuria & 4 & 0.8 \\
Hematuria & 60 & 13 \\
Hemoglobinuria & 34 & 7.6 \\
Leukocyturia & 76 & 16.4 \\
\hline
\end{tabular}

Table 3. Electrolyte abnormalities seen in 461 leprosy patients

\begin{tabular}{lcc}
\hline & $\mathbf{N}$ & $\%$ \\
\hline Sodium & & \\
$\quad$ Hyponatremia $\left(\mathrm{Na}^{+}<135 \mathrm{mEq} / \mathrm{L}\right)$ & 36 & 7.8 \\
$\quad$ Hypernatremia $\left(\mathrm{Na}^{+}>145 \mathrm{mEq} / \mathrm{L}\right)$ & 2 & 0.4 \\
Potassium & & \\
$\quad$ Hypocalemia $\left(\mathrm{K}^{+}<3.5 \mathrm{mEq} / \mathrm{L}\right)$ & 12 & 2.6 \\
$\quad$ Hypercalemia $\left(\mathrm{K}^{+}>5 \mathrm{mEq} / \mathrm{L}\right)$ & 12 & 2.6 \\
\hline
\end{tabular}

being dependent on the immune response of the host [1,2830]. Time of disease was extremely variable, with some cases being detected in the initial phases and others only in the end stages. The classification of the patients, according to the World Health Organization criteria, showed a higher prevalence of the two poles of the disease (tuberculoid and lepromatous leprosy) [1].

High levels of serum creatinine were seen in 40 patients $(8.6 \%)$. Most of these (45\%) had lepromatous leprosy, which suggests that this form of disease has a stronger association with renal damage. These patients had no other disease besides leprosy; so the renal dysfunction could be attributed to leprosy itself or to the drugs administered during its treatment. Leprosy patients with renal injury have a large spectrum of clinical manifestations, which range from asymptomatic disease to classical nephrotic syndrome [7]. In a study performed by Kirsztajn et al. [31], no obvious alteration of renal function was detected in the analysis of serum creatinine. These same authors proposed the dosing hematuria, microalbuminuria and $\beta_{2}$-microglobulin to detect the earliest alterations of renal involvement in leprosy [31]. Acute renal failure can occur in leprosy, the main cause of this being the occurrence of acute tubular necrosis as a result of sepsis observed in the end stages of the disease [7]. Chronic renal failure seems to be more common in leprosy, due to amyloidosis, being an important cause of death in these patients $[8,14,20]$. Amyloidosis is considered to be the main cause of renal failure in patients with leprosy [8]. Nakayama et al. [8] found that $57 \%$ of leprosy patients with amyloidosis presented renal failure and died because of this condition.

Visceral involvement in leprosy has been reported frequently; many organs can be damaged by leprosy [17]. Some studies have demonstrated that renal lesions are the main cause of death in leprosy patients [10]. Organ dysfunction seems to correlate with the quantity of bacilli and/or the presence of other factors, such as associated diseases, amyloid infiltration, concomitant infections, leprosy reactions and side effects of drugs [17]. In our study, we only included patients who had no other disease besides leprosy, and who were only using the drugs of multidrug therapy (rifampicin, dapsone and clofazimine) or drugs for the reactional states (corticosteroids or thalidomide), so that the renal abnormalities found could be attributed to leprosy or to the nephrotoxic effects of these drugs.

Renal injury seems to be common in patients with erythema nodosum leprosum (ENL) [17,18]. Proteinuria and/or hematuria may occur in multibacillary patients or during episodes of ENL, without overt nephritis or nephritic syndrome and with normal histology [17]. Both glomerular and interstitial lesions are known to occur in lepromatous leprosy [20]. The proliferative glomerular lesions are similar to post-streptococcal glomerulonephritis. Patients with leprosy and skin lesions are probably more susceptible to streptococcal skin infection and subsequent renal involvement [20]. The exact pathogenesis of renal lesions in leprosy is still uncertain [18]. Glomerular lesions can be caused by immune-complexes, which develop during the reactional states, mainly in the erythema nodosum leprosum [20,31,32]. This conclusion is supported by clinical and laboratorial findings, such as visualization of immune complexes and a decrease of serum complement in some cases [5,7,21-25]. In our study, the patients had not been submitted to renal biopsy, so that we could not describe the type of renal injury through histological findings. We also could not determine if there was involvement 
Table 4. Clinical and laboratorial data from tuberculoid leprosy and lepromatous leprosy patients

\begin{tabular}{|c|c|c|c|}
\hline & $\begin{array}{l}\text { Tuberculoid Leprosy } \\
\qquad(\mathrm{n}=129)\end{array}$ & $\begin{array}{l}\text { Lepromatous Leprosy } \\
\qquad(n=138)\end{array}$ & Pvalue \\
\hline \multicolumn{4}{|l|}{ Gender } \\
\hline Male & $44(35 \%)$ & $80(58 \%)$ & 0.01 \\
\hline Female & $85(65 \%)$ & $58(42 \%)$ & 0.005 \\
\hline Age (years) & $39.1 \pm 18$ & $39.5 \pm 19$ & 0.44 \\
\hline Time of disease (months) & $16 \pm 24$ & $25 \pm 39$ & 0.01 \\
\hline Time of treatment (months) & $13 \pm 13$ & $15 \pm 17$ & 0.13 \\
\hline Serum creatinine (mg/dL) & $0.8 \pm 2.9$ & $1.4 \pm 2.9$ & 0.008 \\
\hline Serum urea $(\mathrm{mg} / \mathrm{dL})$ & $27 \pm 12$ & $42 \pm 49$ & 0.0003 \\
\hline Renal failure (serum creatinine $\geq 1.4 \mathrm{mg} / \mathrm{dL}$ ) & $7(6 \%)$ & $18(13 \%)$ & 0.02 \\
\hline \multicolumn{4}{|l|}{ Urinalysis } \\
\hline Proteinuria ( $\geq “+" ”)$ & $4(3.1 \%)$ & $23(16.6 \%)$ & 0.0001 \\
\hline Hematuria $(\geq 1 / \mathrm{HPF})$ & $11(8.5 \%)$ & $25(18.1 \%)$ & 0.03 \\
\hline Leukocyturia ( $\geq 5 / \mathrm{HPF})$ & $21(16.2 \%)$ & $25(18.1 \%)$ & 0.74 \\
\hline
\end{tabular}

P value according to the t test and Fischer's Exact Test; Statistically significant at $\mathrm{p}<0.05$ (95\% confidence interval).

Table 5. Association between duration of disease, duration of treatment and high levels of serum creatinine (renal failure) in tuberculoid leprosy and lepromatous leprosy patients

\begin{tabular}{lcc}
\hline & Duration of disease x renal failure & Duration of treatment x renal failure \\
\hline General sample & & \\
OR & 0.48 & 0.84 \\
C & $0.23-0.98$ & $0.41-1.71$ \\
P value & 0.04 & 0.72 \\
Tuberculoid Leprosy & & 3.47 \\
OR & 0.42 & $0.43-90.9$ \\
C & $0.07-2.61$ & 0.36 \\
P value & 0.42 & 0.55 \\
Lepromatous leprosy & 0.24 & $0.18-1.67$ \\
OR & $0.07-0.73$ & 0.36 \\
C & 0.008 & \\
P value & & \\
\hline OR $=$ odds ratio, CI = 95\% mean confidence interval, $\mathrm{p}$ value according to the chi square test; Statistically significant at \\
p < 0.05 (95\% CI).
\end{tabular}

of immune complexes in the pathogenesis of glomerular damage, because the serum complement of these patients had not been analyzed.

Important urinalysis abnormalities were found, such as proteinuria, hematuria, hemoglobinuria and leukocyturia. These abnormalities were more frequent in the lepromatous leprosy patients. When comparing the patients from the two poles of leprosy (tuberculoid and lepromatous), we saw some important differences in renal involvement. There was no significant difference in age or time of treatment. Treatment is usually longer in multibacillary forms $[26,29,30]$. Some of the patients abandoned treatment before it was finished, so that the average time of treatment was not significantly different between these groups. Lepromatous leprosy is usually a chronic disease, with a more indolent course in comparison with the paucibacillary forms $[1,29,30]$. The incidence of renal failure was significantly higher among the lepromatous leprosy patients. The mean serum creatinine and urea levels were also significantly higher in this group of patients. The urinalysis also showed more alterations in these patients, leukocyturia being found in the two groups at the same frequency. Nephrotic levels of proteinuria were only found in lepromatous leprosy patients and in one indeterminate patient, suggesting that this condition occurs only in multibacillary forms. Peter et al. [10] detected urinalysis abnormalities in only one patient among 30 cases. This patient was in the reactional stage and presented hematuria and leukocyturia. None of the patients analyzed in that study presented proteinuria. The biopsy findings of these patients showed nephritis of all kinds in all patients, except in six who had no renal abnormality. Hematuria was a common finding in a study performed in patients from São Paulo, Brazil, where it was found in 21 of 96 patients [31]. Proteinuria was not frequent in these patients, being detected in only two, with one reaching nephrotic levels [31]. The presence of these abnormalities in urinalysis suggests glomerular injury. The clinical features of glomerulonephritis in patients with leprosy most commonly 
consist of asymptomatic hematuria and/or proteinuria [18]. Higher levels of proteinuria, hematuria and nephrotic syndrome are found in lepromatous leprosy patients [7]. Shwe \& Woodruff [24], analyzing 221 leprosy patients, found a significant higher frequency of proteinuria in lepromatous leprosy patients, especially in those with a high bacterial index and those in reactional states.

There was an association between time of disease and high levels of creatinine. There was no association between time of treatment and high levels of creatinine, so the renal injury detected in some of the patients seems to have no relation to the drugs used for treatment. There was no association between time of disease, time of treatment and high levels of creatinine in tuberculoid leprosy patients, which suggests that renal injury is milder in this presentation of leprosy than in lepromatous leprosy. The high numbers of bacilli seen in the lepromatous patients could be the cause of the more frequent and prominent renal injury.

We detected three deaths $(0.6 \%)$, caused by septic shock and pulmonary embolism. In a review of 199 autopsies of leprosy patients, the causes of death were infectious diseases $(37 \%)$, renal failure $(23 \%)$ and cardiovascular diseases $(22 \%)$ [8]. Renal injury was not responsible for any death in our study, which demonstrates the prompt identification of renal function abnormalities and the correct management of this condition in all of these patients.

In summary, renal function abnormalities are common in leprosy, especially in lepromatous leprosy patients. Urinary abnormalities include proteinuria, hematuria, hemoglobinuria and leukocyturia. There was an association between time of disease and high levels of creatinine, which suggests that the longer the duration of the disease the higher the possibility of renal injury. This association was not found in tuberculoid leprosy patients, suggesting that renal involvement in leprosy is related to the quantity of bacilli present in the body. There was no association between the time of treatment and the levels of creatinine; so we can conclude that renal damage is not significantly influenced by the drugs used in the treatment of this disease, even with the use of nephrotoxic agents. It is important to evaluate renal function in all leprosy patients in order to detect any abnormality and prevent renal failure, which is still a potential cause of death in this disease.

\section{Acknowledgments}

We are very grateful to the team of physicians, residents, medical students and nurses of the Walter Cantídio University Hospital for the assistance provided to the patients and for the technical support provided for the development of this research. We also thank Professor José Gomes Bezerra Filho, of the Discipline of Biostatistics, Federal University of Ceará, for statistical support. This research was financially supported by the Brazilian Research Council (Conselho Nacional de Desenvolvimento Científico e Tecnológico - CNPq, Brasil).

\section{References}

1. Britton W.J., Lockwood D.N. Leprosy. Lancet 2004;363:120919.

2. World Health Organization. Elimination of Leprosy as a Public Health Problem - Leprosy Today. In: http://www.who.int/ lep, August 2003.

3. Chugh K.S., Kaur S., Kumar B., et al. Renal lesions in leprosy amongst north India patients. Postgrad Med J 1983;59:70711.

4. Chugh K.S., Sakhuja V. Renal lesions in Leprosy. J Assoc Physicians India 1991;39:163-4.

5. Date A., Johny K.V. Glomerular subepithelial deposits in lepromatous leprosy - microscopic study. Am J Trop Med Hyg 1975;24:853-6.

6. Jain P.K., Kumar S., Govil D.C., et al. Renal changes in Leprosy and its reactions (abstract p.69). In: Abstracts of the X International Congress of Nephrology (London). London, UK, 1987.

7. Nakayama E.E., Ura S., Negrão R.F., et al. Lesões renais em Hanseníase. J Bras Nefrol 1995; 17:148-57.

8. Nakayama E.E., Ura S., Fleury R.N., Soares V. Renal lesions in Leprosy: a retrospective study of 199 autopsies. Am J Kidney Dis 2001;38(1):26-30.

9. Nigam P., Pant K.C., Kapoor K.K., et al. Histo-functional status of kidney in Leprosy. Indian J Lepr 1986;58(4):567-75.

10. Peter K.S., Vijayakumar T., Vasudevan D.M., et al. Renal involvement in Leprosy. Lepr India 1981;53:163-78.

11. Weiner I.D., Northcutt A.D. Leprosy and glomerulonephritis: Case report and review of literature. Am J Kidney Dis 1989;13(5):424-9.

12. Mitsuda K., Ogawa M. A study of 150 autopsies on cases of Leprosy. Int J Lepr 1937;5:53-60.

13. Kean B., Childress M.E. A summary of 103 autopsies on Leprosy patients on the Isthmus of Panama. Int J Lepr 1942;10:51-9.

14. Ahsan N., Wheeler D.E., Palmer B.F. Leprosy-associated renal disease: case report and review of the literature. $\mathrm{J}$ Am Soc Nephrol 1995;5(8):1546-52.

15. Date A., Thomas A., Mathal R., Johny K.V. Glomerular pathology in Leprosy and electron microscopic study. Am J Trop Med Hyg 1977;26:266-72.

16. Mittal M.M., Agarwal S.C., Maheshwari H.B., Kumar S. Renal lesions in Leprosy. Arch Pathol 1972;93:8-12.

17. Klioze A.M., Ramos-Caro F.A. Visceral leprosy. Int J Dermatol 2000;39 (9):641-58.

18. Kirsztajn G.M., Pereira A.B. Comprometimento renal na Hanseníase. In: Cruz J., Barros R.T. (eds.) Atualidades em Nefrologia 4. São Paulo: Sarvier, 1996.

19. Schuttieworth J.S., Ross S.H. Secondary amyloidosis in Leprosy. Ann Intern Med 1956;45:23-38.

20. Singhal P.C., Chugh K.S., Kaur S., Malik A.K. Acute renal failure in leprosy. Int J Lepr 1977;45 (2):171-174.

21. Bullock W.E., Callerame M.L., Panner B.J. Immunohistologic alterations of skin and ultrastructural changes of glomerular basement membranes in Leprosy. Am J Trop Med Hyg 1974; $23: 81-86$

22. Drutz D.J., Gutman R.A. Renal manifestations of Leprosy. Glomerulonephritis - a complication of erythema nodosum leprosum. Am J Trop Med Hyg 1973;22:496-502. 
23. Iveson J.M.L., McDougail A.C., Leathem A.J., Harris H.J. Lepromatous Leprosy presenting with polyarthritis, myositis and immune-complex glomerulonephritis. Br Med J 1975;3:619-21.

24. Shwe T., Woodruff A.W. Renal involvement in leprosy. Trans R Soc Trop Med. Hyg 1972;66(1):26-7.

25. Valles M., Cantarelli C., Fort J., Carrera M. IgA nephropathy in Leprosy. Arch Intern Med 1982;142:1238.

26. Ishii N. Recent advances in the treatment of leprosy. Dermatol Online J 2003;9(2):5.

27. Lockwood D.N.J. Leprosy elimination - a virtual phenomenon or a reality? Br Med J 2002;324:1516-8.
28. Goulart I.M.B., Penna G.O., Cunha G. Imunopatologia da hanseníase: a complexidade da resposta imune do hospedeiro ao Mycobacterium leprae. Rev Soc Bras Med Trop 2002;35(4):365-75.

29. Jacobson R.R., Krahenbuhi J.L. Leprosy. Lancet 1999;353:655-60.

30. Margarido-Marchese L., Tedesco-Marchese A.J., Riviti E.A. Hanseníase. In: Veronesi R., Focaccia R. (eds.) Tratado de Infectologia. São Paulo: Atheneu, 2002.

31. Kirsztajn G.M., Nishida S.K., Silva M.S., et al. Renal abnormalities in Leprosy. Nephron 1993;65:381-4.

32. Shwe T. Immune complexes in glomeruli of patients with Leprosy. Lepr Rev 1972;42:282-9. 